\title{
Creación de la Sociedad Chilena de Neurociencia
}

\section{Estimado Sr. Editor}

La investigación científica en el área de las neurociencias ha tenido un crecimiento exponencial en el mundo durante la última década. Chile goza de una larga tradición en investigación neurobiológica y neurológica, lo que ha resultado en la existencia de un número importante de investigadores interesados en el área. Considerando este desarrollo, no es extraño que el año pasado se haya creado la Sociedad Chilena de N eurociencia; reconociendo el potencial enorme de estudiar el sistema nervioso como un campo separado, pero convergente para ambas visiones (investigación básica y clínica). La primera reunión científica, señalando el compromiso de la comunidad de neurocientistas de crear esta sociedad, se realizó en la Facultad de Ciencias de la Universidad de Chile el 24 de Noviembre del 2004.

La Sociedad Chilena de Neurociencia se crea como una organización independiente sin fines de lucro, dedicada a la promoción de la neurociencia y a la comunicación entre investigadores en diferentes disciplinas, incluyendo biólogos, bioquímicos, médicos, odontólogos y psicólogos, entre otros. Desde su fundación, y con la elección de su primer Directorio en M ayo del 2005, una de sus preocupaciones ha sido establecer vínculos con la Society for N euroscience de los Estados U nidos, Sociedad creada en 1970, y que cuenta con más de 36.000 miembros en la actualidad(1), e IBRO (International Brain Research Organization), creada en 1960, y representando alrededor de 51.000 neurocientíficos provenientes de 111 países en todo el mundo(2).

\section{Situación del desarrollo de la neurociencia en Chile}

La neurociencia básica es una de las áreas de mayor desarrollo en investigación en Chile, estando representada por equipos sólidos y con interacción permanente con centros de investigación internacionales. Sin embargo, el desarroIlo de la neurociencia clínica, tanto en el área de la investigación experimental, como la propiamente clínica, atraviesa un período particularmente difícil. La alta demanda económica y asistencial que predomina en la organización académica de las escuelas de medicina, restringen el apoyo a la investigación realizada por médicos clínicos, lo que se agrava por las dificultades en conseguir financiamiento para investigación, de por sí escasos y muy competitivos. El resultado de lo anterior es una disminución importante de grupos de investigación involucrados en el estudio de patologías neurológicas liderados por un médico clínico. No deja de ser paradójico que, el desarrollo y tradición de la neurobiología en Chile parece ser uno de los elementos que dificulta el desarrollo de investigación en neurología y neurocirugía, al constituirse en competencia para la obtención de recursos ${ }^{(3)}$. La importancia de mantener un espacio para la investigación clínica en neurología y neurocirugía es indiscutible, y va a beneficiar tanto a quienes trabajan en temas esencialmente básicos-experimentales como a quienes están interesados en la investigación en cuadros patológicos. La disminución de médicos trabajando en investigación no es un problema que se limite a la neurociencia, sino que es transversal a muchas disciplinas, y es un problema que afecta 
tanto a Chile como al resto del mundo(4). Sin embargo, y a pesar de las dificultades, es nuestro convencimiento que el trabajo conjunto de neurocientistas básicos y clínicos se asocia a enormes ventajas relacionadas tanto al abordaje conjunto de proyectos de investigación, como para la enseñanza de la medicina y de los especialistas en neurología, neurocirugía, psiquiatría y psicología(5).

\section{Interacción básico-clínica en Chile y en el mundo}

La asociación entre los centros académicos de investigación y científicos a empresas biotecnológicas o de desarrollo médico es una característica predominante en las universidades en los Estados Unidos y Europa. De hecho, esta asociación es un punto importante en la forma como se aborda la investigación en neurociencias tanto para los investigadores científicos puros como para aquellos en el ambiente clínico. La visión a nivel mundial es que, durante la próxima década, podrían generarse descubrimientos señeros en neurociencia, y lo que es más importante, estos descubrimientos podrían traducirse en un beneficio concreto para la salud de las personas. Sin embargo, en Chile, la asociación entre la academia y la empresa biotecnológica o la medicina es un fenómeno de baja frecuencia. Se observan algunas colaboraciones entre los distintos ámbitos, más bien tímidos, que son el resultado de motivaciones y motores personales, más que de políticas institucionales. Obviamente hay mucho camino por recorrer.

Los rápidos cambios sociales y económicos que está sufriendo nuestro país generan un desafío adicional para Chile. En orden de desarrollar la capacidad de responder a estas demandas crecientes, Chile, al igual que el resto de los países en vías de desarrollo, debe favorecer tanto la salud como la educación de sus ciudadanos. La neurociencia puede jugar un papel clave en este desafío. M uchos de los desórdenes del sistema nervioso interfieren tanto con la educación como con la salud, y de esa manera constituyen una verdadera amenaza para el desarrollo económico y so- cial del país. No podemos permitirnos menospreciar la carga social y económica que resulta de ignorar el impacto que tienen los desórdenes del sistema nervioso. A pesar del impacto económico y personal profundo que tienen las alteraciones neurológicas, psiquiátricas, y del desarroIlo, los que incluyen un rango amplio de condiciones inhabilitantes, como la epilepsia, los accidentes vasculares, la esquizofrenia, la depresión, el retardo mental, y las demencias; los sistemas de salud, tanto privados como estatales, y particularmente en los países en vías de desarrollo, les prestan poca atención. Hoy en día, hay un reconocimiento creciente de la prevalencia de estas patologías y de la disponibilidad de estrategias de prevención y tratamientos que hace posible reducir su impacto, incluso si los recursos son limitados ${ }^{(6)}$. La diversidad de estos desórdenes constituye una tarea compleja para los investigadores tanto del ámbito básico como clínico y ponen en manifiesto la necesidad de aunar fuerzas para permitir enfrentarlos en forma conjunta.

\section{Espacio que ofrece la Sociedad Chilena deNeurocienciaala comunidad científica y clínica nacional}

La Sociedad Chilena de N eurociencias acoge a una amplia gama de investigadores científicos dedicados al estudio del sistema nervioso normal y patológico, y a la traducción de este conocimiento en aproximaciones prácticas de su prevención y manejo ${ }^{(7)}$. Las áreas de la neurociencia que convoca la Sociedad Chilena de Neurociencia incluye el estudio del sistema nervioso en su gran diversidad, involucrando el estudio de su desarroIlo, los mecanismos de sensación y percepción, sistema motor, aprendizaje y memoria, sueño, stress, envejecimiento y el estudio de patologías neurológicas y psiquiátricas entre otras. La variedad disciplinaria de sus miembros favorecerá una aproximación interdisciplinaria, usando metodologías múltiples (de la genética, de la biología molecular y celular, de la anatomía, neurofisiológica, de sistemas, comparativa, y del comportamiento) para estudiar el sistema nervioso 
tanto en preparaciones animales como en el ser humano. Pensamos que, con nuestra multidisciplinariedad, podremos facilitar la traducción de este conocimiento fundamental es estrategias reales para el tratamiento de enfermedades del sistema nervioso. Al mismo tiempo, la Sociedad Chilena de Neurociencia promoverá la transferencia de información desde el desempeño clínico hacia el campo de la investigación experimental. De esta forma, podremos contribuir al desarrollo de la neurociencia, favoreciendo la utilización de los recursos disponibles para el estudio de la función del sistema nervioso en salud y enfermedad.

Con relación a esta gama amplia de estudios y aproximaciones, la Sociedad se plantea entre sus objetivos la promoción del conocimiento del sistema nervioso mediante la integración de investigadores en las diversas áreas, facilitando la integración de la investigación dirigida a todos los niveles de la organización biológica, y favorecien-

\section{Referencias}

1. Society for Neuroscience: http://www.sfn.org/

2. International Brain Research Organization (IBRO): http://www.ibro.org/

3. Fadic, R. Investigación en Neurociencias Clínicas. Rev Chil Neuro-Psiquiat. 2004; 42: 159-162.

4. Rosenberg LE. The physician-scientist: an essential -and fragile link in the medical research chain. J Clin Invest. 1999; 103: 1621-1626.

5. Zemlo TR, Garrison HH, Partridge NC, Ley TJ. do la aplicación de la investigación y el conocimiento generado en el entendimiento de las enfermedades y eventualmente en el desarrollo y mejora de tratamientos para ellas. Para ello, se organizarán actividades que promuevan el desarrollo profesional, el acceso a información, y actividades educativas para neurocientistas a lo largo de su carrera, desde el pregrado hasta el postdoctorado, favoreciendo una mayor participación de científicos provenientes de todo el país. Además, considerando el impacto positivo que la neurociencia más allá del mundo académico, la Sociedad se propone promover la información y educación del público en general con relación a los descubrimientos científicos y su importancia, e informar a los legisladores y líderes sobre la generación del conocimiento científico en el área de la neurociencia, y su importancia en el desarrollo de políticas públicas, beneficios para la sociedad e iniciativas de promoción del desarrollo científico.
ThePhysician-Scientist: career issues and challenges at the year 2000. FASEB J. 2000; 14: 221-230.

6. Committee on Nervous System Disorders in Developing Countries, Board on Global Health Neurological, Psychiatric, and Developmental Disorders: M eeting the Challenge in the Developing World National Academies Press. ISBN: 0-30957008-5, (2001). Disponible en URL: http://www. nap.edu/catalog/10111.html.

7. Sociedad Chilena de Neurociencia: http://www. socneurociencia.cl/

Correspondencia:

Rommy von Bernhardi M.

Presidenta de la Sociedad Chilena de

Neurociencia

Departamento N eurología, Facultad de M edici-

na, Pontificia Universidad Católica de Chile.

E-mail: rvonb@med.puc.cl 